\title{
A Fuzzy C-Means Algorithm for Fingerprint Segmentation
}

\author{
Pedro M. Ferreira, Ana F. Sequeira, and Ana Rebelo \\ INESC TEC \\ $\{$ pmmf, afps, arebelo\}@inesctec.pt
}

\begin{abstract}
Fingerprint segmentation is a crucial step of an automatic fingerprint identification system, since an accurate segmentation promote both the elimination of spurious minutiae close to the foreground boundaries and the reduction of the computation time of the following steps. In this paper, a new, and more robust fingerprint segmentation algorithm is proposed. The main novelty is the introduction of a more robust binarization process in the framework, mainly based on the fuzzy C-means clustering algorithm. Experimental results demonstrate significant benchmark progress on three existing FVC datasets.
\end{abstract}

Keywords: fingerprint segmentation, fuzzy C-means clustering, morphological processing

\section{Introduction}

Fingerprint recognition systems have been developed to be used in a wide range of personal identification domains, such as civil identification, access control and forensics. A fingerprint recognition system is composed by several processing steps: from fingerprint segmentation to minutiae extraction and matching [1]. A fingerprint image is usually composed by two distinct regions: the foreground and the background. The foreground area, which contains effective ridge information, is originated from the contact of a fingertip with the sensor. The noisy area at the border of the image, with no effective information, corresponds to the background. The separation of the content into these two groups allows to: 1) remove spurious minutiae located at the boundary of the foreground; and 2) speed up the following tasks by restricting the processing to the foreground area, making the fingerprint segmentation one of the most relevant steps of an automatic fingerprint recognition system [1].

Several fingerprint segmentation methods have been proposed. Bazen et al. [2] suggested a pixel-wise method, in which three features (coherence, mean and variance) are computed for each pixel and then a linear classifier associates the pixel with the foreground or the background. The method presented by Chen et al. [3] uses a block cluster degree along with a linear classifier. Wu et al. [4] proposed a fingerprint segmentation method based on the Harris corner detector. The image points with the strongest Harris response are considered as foreground points and the final segmentation is obtained as their convex hull. Liu 


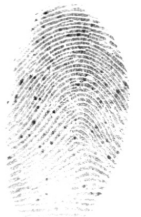

(a)

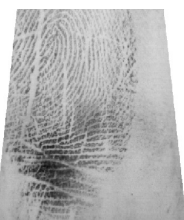

(b)

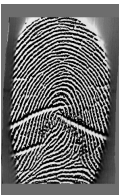

(c)

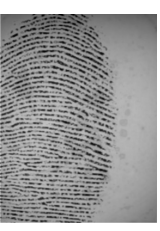

(d)

Fig. 1: Sample images of FVC2004 database. The sensors used in (a), (b), (c), and (d) are low-cost optical sensor, low-cost capacitive sensor, optical sensor, and synthetic generator, respectively.

et al. [5] suggested a classification using the AdaBoost classifier with two novel features (block entropy and block gradient entropy) and several commonly used features (coherence, mean, variance and Gabor features). More recently, Fahmy et al. [6] proposed a fingerprint segmentation method based on morphological operations. A range filter is applied to the fingerprint image followed by an adaptative thresholding binarization. A final morphological post-processing step is performed in order to remove holes in both foreground and background.

Fingerprints acquired by different sensors display different background noise as may be observed in Fig. 1. Most of the available segmentation algorithms demonstrate lack of adaptability to this variability of noise, which increases errors in the fingerprint recognition process.

In this paper, we proposed an extension of the algorithm presented by Fahmy et al. [6] by introducing a new binarization process in the framework. Our segmentation method overcomes the problems inherent to the image capture with various sensors. The effectiveness of the proposed algorithm is validated by experiments performed on all available databases of the Fingerprint Verification Competition (FVC). ${ }^{1}$ The satisfatory results of this method will make possible to address a challenge posed by the Portuguese Mint and Official Printing Office (INCM), that often uses and promotes the R\&D of the country.

The paper is organized in four sections including the Introduction (Section 1). In Section 2, the proposed fingerprint segmentation method is fully described. Section 3 reports the experimental results. Finally, conclusions and topics for future work are presented in Section 4.

\section{Proposed Fingerprint Segmentation Method}

As already mentioned, the baseline for the methodology proposed in this paper follows the morphological fingerprint segmentation algorithm presented by Fahmy et al. [6]; throughout this paper the algorithm of Fahmy et al. [6] will be referred as the baseline method. The main novelty of the proposed method

\footnotetext{
${ }^{1}$ https://biolab.csr.unibo.it
} 


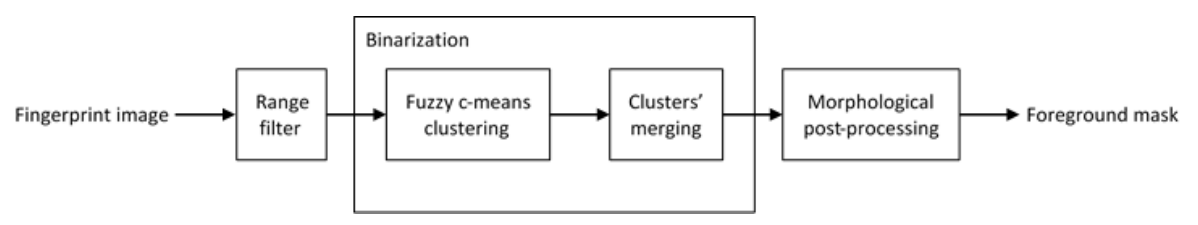

Fig. 2: Architecture of our proposed framework for fingerprint segmentation.

is related to the involved binarization process. While in the baseline method [6] a simple adaptative thresholding binarization is used, we suggest a more robust binarization method by using a Fuzzy C-means (FCM) approach. Since fingerprint images are frequently contaminated by noise (e.g, produced by the sensors) our approach is capable of coping with noise based on the clusters association of the FCM algorithm.

The high-level operations that compose our algorithm are presented in Fig. 2. First, a block-wise range filter is applied to the grey-scale fingerprint image in order to enhance the ridges. Afterwards, the resulting range image is binarized using the FCM algorithm along with a clusters' merging procedure. Finally, a set of morphological operations is applied to the binary image in order to compute the final foreground mask. Next, the several steps will be explained in more detail.

\subsection{Range Filter}

The problem of fingerprint segmentation is tightly coupled with the extraction of features, especially when it comes to the development of segmentation methods with sensor interoperability. The proposed methodology uses the range image as a feature. As demonstrated by Fahmy et al. [6], the range image provides better performance and interoperability capabilities than entropy and entropy-gradient features.

The range filter is a non-linear filtering method that replaces the original grey level of a pixel by the range value of the grey levels of the pixels in a specified neighbourhood:

$$
\begin{aligned}
R(i, j)=\max \{I(m, n) \mid(m, n) \in w(i, j)\} & \\
& -\min \{I(m, n) \mid(m, n) \in w(i, j)\}
\end{aligned}
$$

where $I$ is the input image, $R$ is the output image, and $w$ represents the neighbourhood centred at image coordinates $(i, j)$. The resulting range image is illustrated in Fig. 3b, in which the boundaries of the ridges are clearly enhanced.

\subsection{Binarization}

The binarization process is composed by two main steps: 1) the range image is clustered using the FCM algorithm and then 2) a clusters' merging procedure is applied in order to build up the final foreground mask. 


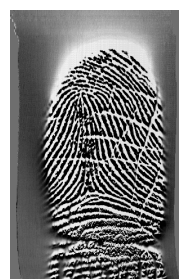

(a)

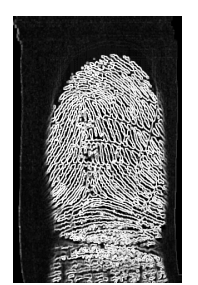

(b)

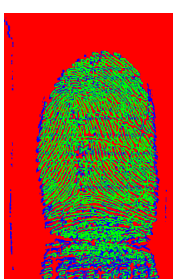

(c)

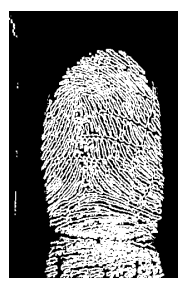

(d)

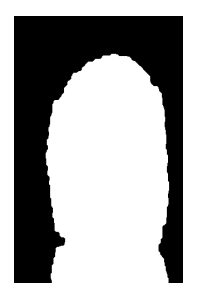

(e)

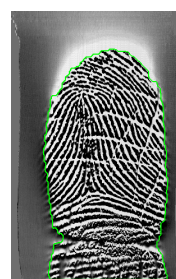

(f)

Fig. 3: Outputs of the proposed fingerprint segmentation method: (a) Original image, (b) Range filter response, (c) FCM clustering of image (b) for $C=3$, (d) Binary image, (d) Final foreground mask, and (d) Foreground boundary superimposed on the original image.

FCM clustering FCM is a clustering algorithm in which a sample can be associated into more than one cluster with different degrees of similarity [7]. Let $X=\left(x_{1}, x_{2}, \ldots, x_{N}\right)$ denotes an image with $N$ pixels to be partitioned into $C$ clusters, where $x_{j}$ represents the data samples, with $j=1, \ldots, N$. The goal is to find an optimal fuzzy $C$-partition that minimizes the following objective function:

$$
J(U, V ; X)=\sum_{j=1}^{N} \sum_{i=1}^{C} u_{i j}^{m}\left\|x_{j}-v_{i}\right\|^{2}
$$

where $v_{i}$ is the centroid of the $i^{t h}$ cluster, $u_{i j}$ is the membership function of pixel $x_{j},\|\cdot\|$ represents the Euclidean distance, and $m$ is a weighting exponential parameter on each fuzzy membership with $1 \leq m<\infty$.

Although the fingerprint area is essentially composed by two distinct objects, namely the ridges (dark pixels) and the valleys (bright pixels), the FCM algorithm is used in order to cluster the range image into three clusters $(C=3)$. The underlying assumption is that one of the clusters will be composed by pixels with high probability of being foreground, another one by pixels with high probability of being background, and a third one by pixels where the decision is not sufficiently reliable. The advantage of partitioning the range image into three clusters rather than just two is to avoid misclassified pixels especially in low quality fingerprint images. Hence, after this, a clusters' merging procedure is used in order to classify each cluster as foreground or background.

Clusters' merging procedure Given the resulting clusters $C_{i}, i=1, \ldots, 3$, and the mean $\overline{C_{i}}$ intensity of each cluster in the range image $R$, the clusters $C_{i}$ are classified as foreground and background as follows:

- the cluster with the highest mean intensity is considered as foreground. Intuitively, pixels with higher range filter response belong to ridges and, hence, should be considered as foreground (green pixels of Fig. 3c). 
- the cluster with the lowest mean intensity is considered as background. The assumption is that pixels with lower range filter response belong to valleys or noise and, hence, should be classified as background (red pixels of Fig. $3 c)$.

- the remaining cluster (blue pixels of Fig. 3c) is selected for further analysis.

The remaining cluster is composed by pixels of both foreground and backgound classes. To find the resultant classification it is important to know the relation among the pixels. In doing so, a connected component analysis is performed. Each connected region is considered as foreground or background according to two criteria:

Let $R_{i}$ be the $i^{\text {th }}$ connected region and $R_{d_{i}}$ be its dilated region.

1. The neighbourhood-based criterion is defined by:

$$
\frac{A\left(R_{d_{i}} \cap \text { foreground }\right)}{A\left(R_{d_{i}}\right)}>T_{1}
$$

where $A$ denotes the area in pixels and $T_{1}$ is a threshold experimentally obtained. In this manner, a connected region $R_{i}$ is classified as foreground if it is in the neighbourhood of pixels already classified as foreground.

2. The variance-based criterion is defined by:

$$
\frac{\left|\sigma^{2}\left(R_{i}\right)-\sigma^{2}\left(R_{d_{i}}\right)\right|}{\max \left(\sigma^{2}\left(R_{i}\right), \sigma^{2}\left(R_{d_{i}}\right)\right)}>T_{2}
$$

where $\sigma^{2}(\cdot)$ denotes de variance of the grey-levels within the specified region and $T_{2}$ is a threshold experimentally obtained. This condition guarantee that connected regions with high grey level differences to their neighbouring-pixels are not excluded.

Afterwards, the foreground region is computed as the union of all the connected components $R_{i}$ which met at least one of the conditions previously described. The final result of this step is illustrated in Figure 3d.

\subsection{Morphological Post-processing}

At this stage the binary foreground mask is composed by several disjoint binary regions with ragged boundaries. Therefore, a post-processing is required in order to obtain the final foreground mask. The post-processing operations include: 1) a morphological closing filter in order to join small adjacent regions; 2) a region filling algorithm to fill interior holes of the binary objects; 3) a morphological opening filter to eliminate thin protrusions and, generally, smooth the contours; and 4) the largest binary component of the image is selected and assumed as the final foreground mask. The result of the morphological post-processing is illustrated in Figure 3e. 


\section{$3 \quad$ Experimental Results}

The main goal of fingerprint segmentation is to remove the noisy background from the images and, hence, increase the overall accuracy of an automatic fingerprint recognition system. In this regard, the quantitative evaluation of the proposed fingerprint segmentation algorithm was made in two steps: (i) a comparison of the automatic segmentation results with ground-truth data (obtained by manually segmenting fingerprint images); and (ii) a goal-directed performance evaluation, in order to assess the overall accuracy of a fingerprint recognition system that integrates the proposed segmentation method.

In both evaluations, the proposed fingerprint segmentation algorithm was directly compared with other three state-of-the-art algorithms: 1) the baseline segmentation algorithm [6];2) the fingerprint segmentation method of the MINDTCT module from NBIS [8]; and 3) a well known variance-based method presented by Kovesi [9].

Experiments were conducted using the public FVC databases: FVC2000, FVC2002 and FVC2004. These databases include fingerprint images acquired with several sensor types (i.e. optical, capacitive and thermal sweeping sensors). Each of them contains four databases, namely DB1_A, DB2_A, DB3_A and DB4_A, composed by 800 fingerprint images acquired from 100 persons with 8 fingerprints per person. The parameters used by the proposed segmentation method were the same in all the experiments. The FCM algorithm used $m=2$ and the thresholds of the clusters merging procedure were fixed as $T_{1}=0.8$ and $T_{2}=0.2$.

\subsection{Ground-truth based evaluation}

In this experiment 240 images from the three FVC databases were randomly selected. It includes 20 images of each subset in order to guarantee the evaluation in images acquired from different sensor types.

The manual segmentation of the fingerprint images was performed by three specialists in fingerprint recognition. The segmentation error rate $(E r r)$ used to quantify the boundary differences, between the manual segmentation $(G T)$ and the automatic segmentation $(A S)$, is defined as:

$$
E r r=\frac{\#(G T \oplus A S)}{\# G T} \times 100
$$

where $\oplus$ represents the exclusive-OR operation. Err is a measure of the misclassified pixels in the foreground and the background. Table 1 presents the average

Table 1: The comparison of the 4 segmentation methods in terms of Err.

\begin{tabular}{|l||c|}
\hline & Average $\operatorname{Err}(\%)$ \\
\hline Proposed & $\mathbf{6 . 5 0}$ \\
Fahmy et al. [6] (baseline) & 7.47 \\
NBIS [8] & 11.50 \\
Kovesi [9] & 15.05 \\
\hline
\end{tabular}



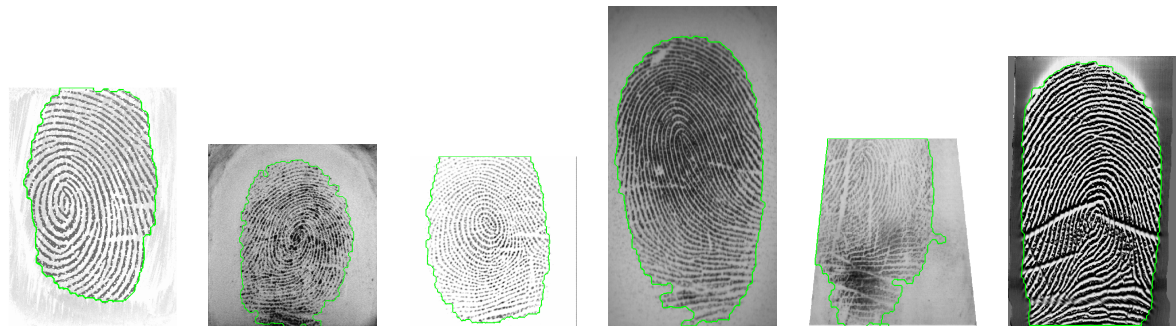

Fig. 4: Results of the proposed fingerprint segmentation algorithm in images of the FVC databases aquied from different sensors types.

Err of the three specialists for each segmentation method involved in the comparison. The proposed segmentation method outperformed the three state-of-art segmentation methods. Fig. 4 shows the segmentation of some images using the proposed technique.

\subsection{Goal-directed performance evaluation}

In this section, a goal-directed performance evaluation that assesses the overall improvement in the system performance that incorporates the proposed segmentation method is presented. Such evaluation is capable of providing a more reliable assessment of the performance benchmark and is directly associated with the ultimate goal of the system. In this regard, an in-house robust fingerprint verification system was built up and the proposed fingerprint segmentation method was compared with other three state-of-the-art methods by replacing the segmentation module of their system.

Table 2 summarizes the results in all the 9600 images of the FVC databases in terms of $E E R$ (equal-error rate). A first observation is that the in-house fingerprint verification system achieves, in general, better results when it uses the proposed segmentation algorithm rather than the other three state-of-the-art methods. More concretely, the performance of the fingerprint verification system is improved in 7 of the 12 FVC databases when our segmentation method is integrated in the system. Likewise, the proposed fingerprint segmentation method promotes the lowest average EER (3.57\%) and the lowest standard deviation $(1.87 \%)$. These results indicate that the proposed segmentation algorithm out-

Table 2: The comparison of the segmentation methods in terms of $E E R(\%)$. Numbers in boldface are the best results.

\begin{tabular}{|c|c|c|c|c|c|c|c|c|c|c|c|c|c|}
\hline \multirow[b]{3}{*}{ Drent } & \multicolumn{3}{|c|}{ FVC2000 } & \multicolumn{4}{|c|}{ FVC2002 } & \multicolumn{4}{|c|}{ FVC2004 } & \multirow[t]{2}{*}{ Average } & \multirow[t]{2}{*}{ Stdev } \\
\hline & DB1 1 DB2 & DB3 & DB4 & DB1: & DB2 & DB3 & DB4 & DB1 & DB2 & DB3 & DB4 & & \\
\hline & \begin{tabular}{|l|l|}
2.64 & $\mathbf{1 . 1 8}$ \\
\end{tabular} & 4.61 & 3.14 & 1.72 & 0.71 & 5.29 & 2.11 & 5.71 & 5.71 & 5.50 & 4.47 & 3.57 & 1.87 \\
\hline Fahmy et al. & \begin{tabular}{|l|l|l}
4.64 & 1.27
\end{tabular} & 4.79 & 3.29 & 1.61 & 0.82 & 5.36 & 2.18 & 5.90 & 7.75 & 5.61 & 4.64 & 3.84 & 1.98 \\
\hline Kovesi [9] & \begin{tabular}{l|l|l}
2.07 & 1.29
\end{tabular} & 11.03 & 3.24 & 1.47 & 0.91 & 7.43 & 1.96 & 5.74 & 5.79 & 7.84 & 4.39 & 4.43 & 3.19 \\
\hline NBIS [8] & \begin{tabular}{|l|l|l|}
$\mathbf{2 . 0 4}$ & 1.33
\end{tabular} & 11.07 & 3.29 & 1.52 & 1.01 & 7.60 & 2.07 & 5.46 & 6.43 & 11.29 & 4.54 & 4.80 & 3.65 \\
\hline
\end{tabular}


performs the existing methods and can handle better with the sensor interoperability problem. When compared with the baseline algorithm, the proposed segmentation method promoted an overall improvement in the system performance.

\section{Conclusions}

The purposes of segmentation in the fingerprint recognition context are both the reduction of the computation time of the following processing steps and, most importantly, the exclusion of many spurious minutiae located at foreground boundaries. In this paper, a new fingerprint segmentation algorithm was proposed taking as baseline the method presented by [6]. The main contribution was the introduction of a more robust binarization process in the framework. The binarization process comprises two steps: 1) a FCM clustering, followed by 2) a clusters' merging procedure in order to build up the final foreground mask.

Experimental results suggest that the proposed segmentation method outperforms other existing methods in both segmentation error rate and overall improvement in the fingerprint system recognition performance.

\section{References}

1. Maltoni, D., Maio, D., Jain, A.K., Prabhakar, S.: Handbook of Fingerprint Recognition. 2 edn. Springer Professional Computing (2009)

2. Bazen, A.M., Gerez, S.H.: Segmentation of fingerprint images. In: ProRISC 2001 Workshop on Circuits, Systems and Signal Processing. (2001) 276-280

3. Chen, X., Tian, J., Cheng, J., Yang, X.: Segmentation of fingerprint images using linear classifier. EURASIP J. Appl. Signal Process. (January 2004) 480-494

4. Wu, C., Tulyakov, S., Govindaraju, V.: Robust point-based feature fingerprint segmentation algorithm. In Lee, S.W., Li, S., eds.: Advances in Biometrics. Volume 4642 of Lecture Notes in Computer Science. Springer Berlin Heidelberg (2007) 1095-1103

5. Liu, E., Zhao, H., Guo, F., Liang, J., Tian, J.: Fingerprint segmentation based on an adaboost classifier. Frontiers of Computer Science in China 5(2) (2011) 148-157

6. Fahmy, M., Thabet, M.: A fingerprint segmentation technique based on morphological processing. In: Signal Processing and Information Technology(ISSPIT), 2013 IEEE International Symposium on. (Dec 2013)

7. Suri, J.S., Wilson, D.L., Laxminarayan, S.: Handbook of Biomedical Image Analysis - Volume II: Segmentation Models Part B. Kluwer Academic/Plenum Publishers (2005)

8. Watson, C.I., Garris, M.D., Tabassi, E., Wilson, C.L., Mccabe, R.M., Janet, S., Ko, K.: User's guide to NIST biometric image software (NBIS) (2007)

9. Kovesi, P.: Matlab and octave functions for computer vision and image processing. http://www.csse.uwa.edu.au/ pk/Research/MatlabFns/\#fingerprints 